\title{
Oxyurose à Syphacia \\ dans un élevage de Rats de laboratoire
}

\author{
par E. ROMAN et M.-A. POTHIER
}

(Laboratoire de Parasitologie de la Faculté de Médecine et de Pharmacie de Lyon)

\begin{abstract}
Résumé
Pendant l'été 1967, un élevage de rats de laboratoire, à Lyon, semble avoir été en totalité infesté par l'Oxyuride Syphacia muris.

L'évolution de ce Nématode est analogue à celle d'autres espèces de la même famille; elle s'effectue, en grande partie, dans le caecum de l'hôte.

De même, notamment, que celles d'Enterobius vermicularis de l'homme, les femelles de $S$. muris peuvent ramper contre la paroi du gros intestin pour atteindre l'anus.

L'oxyurose du rat, due à cette dernière espèce, évolue d'une manière continue, comme si les femelles de ce Nématode pondaient tous les jours autour de cet orifice.

Les rats jeunes et adultes contractent la parasitose par cohabitation ; il est très peu probable que des larves venant d'éclore pénètrent dans leur organisme par l'anus et remontent ensuite l'intestin postérieur.

Au cours de notre observation, des Syphacia ont pénétré au moins sept fois chez chacun de nos rats; ces Rongeurs se sont ensuite déparasités, comme si l'évolution dans leur organisme de quelques générations de ces helminthes leur conférait l'immunité.
\end{abstract}

\section{Summary}

In Lyon (Rhône-France), during Summer 1967, the A.A. found all the rats of a laboratory breeding infested with the Oxyurid Syphacia muris.

The evolution of this Nematode is very similar to those of other species of the same family which run most of their usual course in the host caecum.

As it occurs with human Enterobius vermicularis, females of $S$. muris often creep along the walls of the large intestine before reaching the anal aperture. 
Rat's oxyurosis, owing to this species, develops regularly, just as if the females every day layed down their eggs all around the anus.

Young and adult rats get infested through cohabitation. There is little opportunity for new hatched larvae to penetrate in the body by the anal aperture and then reach the posterior end of the intestine.

The A.A. observed at least seven times the penetration of Syphacia in rats; but after several generations, the rats probably acquired immunity when they spontaneously get rid of their infestation.

Alors que des Oxyurides s'observent souvent chez les souris de laboratoire, nous n'en avions jusqu'à présent jamais observé chez les Rats albinos issus de Rattus norvegicus (Berkenhout) des élevages de la Faculté de Lyon.

En vérifiant l'infestation d'un Rat d'environ trois mois ( $\left.\mathrm{n}^{\circ} 752\right)$ servant à l'entretien d'une souche de Strongyloides ratti Sandground, nous avons trouvé dans ses déjections, le 29 juillet 1967, en plus de larves rhabditoïdes de ce Nématode, deux femelles de Syphacia. D'après ses caractères et aussi d'après ceux d'individus recueillis ultérieurement lors d'autopsies, nous avons déterminé ces Oxyurides $S$. muris Yamaguti, dont l'hôte principal est Rattus norvegicus, mais qui peut occasionnellement infester la souris, en raison de la brièveté du spicule ( 30 à $36 \mu)$ et de la grande longueur du prolongement caudal (111-129 $\mu)$ chez le mâle et aussi à cause de la taillé réduite des œufs (64-76 $\mu \times 23-32 \mu$, sur 20 œufs, dont dix intrautérins et dix obtenus par prélèvement anal).

S. muris a tout d'abord été trouvé au Japon (S. Yamaguti, 1935). K. L. Hussey $(1956,1957)$ le mentionne de différentes régions des Etats-Unis et du Canada. Il semble avoir toujours été récolté chez des Rongeurs de laboratoire. Nous sommes probablement les premiers à signaler en Europe l'infestation de Rattus norvegicus par cette espèce. Des Syphacia parasitant Rattus rattus (L.) ont été mentionnés de cette partie du Monde. Faute d'avoir obtenu des mâles provenant de cet hôte, il n'est pas possible de départager ceux qui, comme J. D. Tiner (1948), pensent qu'ils doivent être rapportés à $S$. muris et ceux qui, comme l'un de nous (1951), supposent qu'ils appartiennent à une espèce différente.

W. Stahl (1961, 1963), confirmant d'après.K. L. Hussey (1957) les constatations de M. J. R. Prince (1950), a montré que le cycle évolutif de Syphacia muris est direct, comme celui de $S$. obvelata, (Rud.), qui parasite principalement la Souris, élucidé par K. F. Ohan (1952).

Contrairement à ce qu'a signalé G. A. Noble (1965) chez $S$. citelli Tiner et Raousch, trouvé chez un spermophile américain, M. J. R. Prince (1950), K. L. Hussey (1956, $1957)$ et W. Stahl $(1961,1963)$ ont constaté que, comme celles d'Enterobius vermicularis (L.) chez l'homme et celles de Syphacia obvelata chez la souris, les femelles de $S$. muris viennent déposer leurs œufs au niveau de l'anus de leur hôte. Ces deux derniers auteurs ont pu facilement déceler ces éléments de dissémination par la méthode de 
Graham à la cellophane adhésive. Nous avons appliqué cette technique à nos rats sans anesthésie. L'examen des préparations ainsi obtenues a montré beaucoup d'œufs à embryon peu évolué et parfois quelques coques vides.

Après avoir décelé de cette manière des œufs de Syphacia chez le rongeur 752, nous avons fait la même constatation sur plusieurs animaux des deux sexes, âgés de trois mois et moins. Tous nous ont paru supporter leur infestation sans en être incommodés ; aucune irritation au niveau de l'anus n'a été observée. L'évolution de l'oxyurose de l'homme étant discontinue, nous avons jugé intéressant de suivre celle de la parasitose du rat à $S$. muris, en pratiquant quatre fois par semaine pendant deux mois, à partir du début d'août et jusqu'à leur autopsie, des prélèvements anaux sur quatre de nos animaux, qui, au début de l'observation étaient l'un $\left(\mathrm{n}^{\circ} 750\right)$ âgé de près de trois mois, les autres ( $\mathrm{n}^{\circ \mathrm{s}} 751,752$ et 753) seulement de huit semaines. Chez tous, des œufs d'Oxyurides, de un à plusieurs centaines, ont été constatés à tous les examens ; chez un seul, le $\mathrm{n}^{\circ} 752$, trois prélèvements non successifs ont été négatifs (voir les graphiques I et II, qui concernent respectivement les rats 752 et 753 ).

De tels résultats montrent que, contrairement au cas évoqué plus haut, le parasitisme par Syphacia muris chez le rat paraît se manifester extérieurement d'une manière continue; il semble toutefois qu'au cours d'une grande partie de la période d'observation, nos animaux étaient peu infestés et qu'ils n'ont présenté que de courtes poussées d'oxyurose vraiment intense. En admettant, comme l'a établi W. Stahl (1963), que la durée de vie des femelles de cette espèce ne dépasse pas dix jours, au moins sept générations de $S$. muris se sont succédées chez les quatre rats, que nous avons ainsi observés.

Normalement, des œufs du Nématode envisagé ici ont dû être émis à l'anus tous les jours. Nous pensons que les femelles pondent habituellement pendant la nuit et quittent très vite l'hôte; les prélèvements anaux effectués de jour n'ont ramené que deux fois des femelles gravides, une chez le rat 751, une autre chez un animal $\left(\mathrm{n}^{\circ} 746\right)$, dont il sera question plus loin; des examens directs d'anus de rats effectués en fin d'après-midi, vers 19 heures (heure légale), ne nous en ont pas révélé.

Nous n'avons pas pu connaître la source de contamination par des Oxyurides de nos rats de laboratoire. Nous admettons que la cohabitation dans une même cage de rongeurs sains et d'animaux parasités a répandu l'infestation dans tout l'élevage. Nous l'avons vérifié, notamment par l'autopsie, après contact avec un de ces derniers, chez deux animaux adultes de plus de six mois ( ${ }^{\circ *} 746$ et 747), reconnus auparavant indemnes d'Oxyurides et provenant d'une cage, où se trouvaient avec eux, trois congénères non parasités. Nous pensons que les souris élevées à leur voisinage ne doivent pas être incriminées. En novembre, elles n'hébergeaient que Syphacia obvelata; les prélèvements anaux, effectués à cette époque sur 20 d'entre elles, ont été positifs cinq fois $(25 \%)$; le maximum d'œufs décelé sur une préparation était seulement de sept.

Les six rats 746, 747, 750, 751, 752 et 753, parasités par $S$. muris, ont été autopsiés en octobre. Les principales constatations ainsi faites sont résumées dans un tableau, dont les caractères typographiques sont expliqués ci-après. Tous les résultats négatifs sont indiqués par le signe - - Les Oxyurides, recherchés sous leurs différentes formes, 

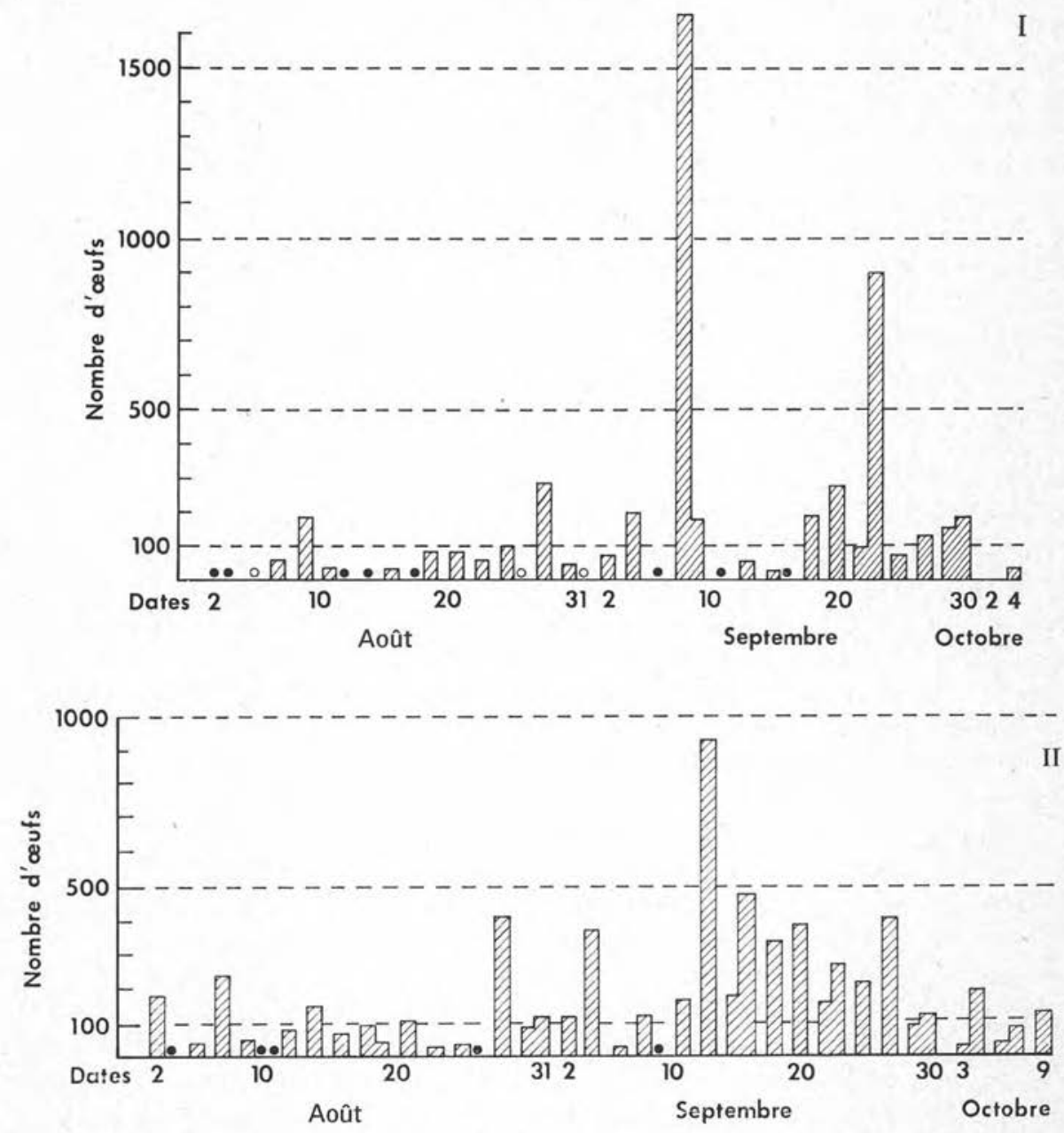

GRAPHIQUES I et II. - Importance des pontes journalières de Syphacia muris chez deux rats albinos du 2 août au début d'octobre 1967. Les longueurs des rectangles verticaux hachurés sont proportionnelles aux nombres d'œufs décelés au prélèvement anal. Les cercles noirs correspondent aux jours où il a été trouvé moins de quinze œufs, les cercles évidés, ceux où il n'en a pas été rencontré. I: rat 752 ; II : rat 753

sont distingués comme suit : mâle ${ }^{\star}$, femelle gravide $q \mathrm{G}$, femelle jeune $\uparrow \mathrm{J}$, larve L, œuf $\omega$. Chez tous, après prélèvement au niveau de l'anus (A.), l'intestin postérieur, en l'espèce les deux derniers tiers du gros intestin (IP), reconnu vide, sinon débarrassé des déjections encombrantes, a été, après ouverture, examiné sous la loupe binoculaire, puis placé jusqu'au lendemain dans du sérum physiologique, qui a alors été centrifugé. En ce qui concerne le caecum, début du côlon compris (C), la paroi a été traitée de 
même ; dans le contenu, les Oxyurides adultes ont été recherchés, soit directement, soit par la méthode des sédimentations et des décantations successives; les larves l'ont été par un procédé d'enrichissement mis au point par l'un de nous (1957). Chez quelques rats, la paroi et le contenu de l'estomac (E) et de l'intestin grêle, coupé en deux tronçons (G1 du côté du pylore, G2 du côté du caecum), ont été soumis à des épreuves analogues.

TABLEAU

Répartition chez quelques rats des divers stades de Syphacia muris au moment de leur autopsie

\begin{tabular}{|c|c|c|c|c|c|c|c|}
\hline Rat & Date & E & G 1 & G 2 & C & IP & A \\
\hline 750 & $12-10$ & & - & - & $2 \% \mathrm{~J}$ & - & $78 \omega$ \\
\hline 751 & $2+10$ & & & & $4 \% G$ & $6 q \mathrm{G}$ & $\begin{array}{r}86 \omega \\
1 \% G\end{array}$ \\
\hline 752 & $4-10$ & & & & $\begin{array}{c}5 \% \mathrm{G} \\
1 \delta^{*}\end{array}$ & $1 \% \mathrm{G}$ & $15 \omega$ \\
\hline 753 & $9-10$ & & & & $\begin{array}{c}14 \% \mathrm{G} \\
1 \% \mathrm{~J} \\
10\end{array}$ & - & $128 \omega$ \\
\hline 746 & $18-10$ & - & - & $2 \% \mathrm{G}$ & $\begin{array}{c}46 \% \mathrm{G} \\
32 \% \mathrm{~J} \\
5 \% \\
2 \mathrm{~L}\end{array}$ & - & - \\
\hline 747 & $16-10$ & $1 \omega$ & $1 \mathrm{~L}$ & - & $\begin{array}{c}1 \% \mathrm{~J} \\
1 \mathrm{~L}\end{array}$ & $\begin{array}{l}1 \% \mathrm{G} \\
1 \uparrow \mathrm{J}\end{array}$ & $162 \omega$ \\
\hline
\end{tabular}

D'après ces constatations, des individus gravides de Syphacia muris ont été récoltés dans le cæcum $s$. $l$. de la plupart des animaux du tableau; l'un de ceux, qui n'en hébergeait pas dans cet organe, en présentait plus près de l'anus; chez le Rongeur le plus parasité, un petit nombre de telles femelles a aussi été décelé dans la dernière portion du grêle. Dans l'intestin postérieur, il en a été vu trois immédiatement après la mort de l'hôte ; l'une d'elles rampait contre la paroi dans la direction de l'anus, une autre nous a paru immobile en position transversale près de cet orifice, comme si elle attendait le moment de s'échapper. Ce comportement nous a paru analogue à celui des individus gravides d'Enterobius vermicularis, tels qu'ils s'observent chez l'homme à la rectoscopie. Une femelle de Syphacia à ce stade se trouvait au niveau de l'anus d'un de nos rats le jour de son autopsie.

Nous avons rencontré moins de femelles jeunes du même Oxyuride, mais les animaux envisagés ci-dessus n'en hébergeaient pas tous dans leur caecum; cependant, une a été décelée dans l'intestin postérieur.

Des mâles n'ont été constatés que dans le caecum et chez trois seulement de nos rats. 
Des œufs n'ayant pas atteint le stade infestant ont été décelés lors de leur autopsie au niveau de l'anus chez presque tous nos Rongeurs; celui, chez qui il n'en a pas été constaté autour de cet orifice, n'hébergeait pas de femelles gravides dans son intestin postérieur. Un œuf avec embryon entièrement développé a été vu dans l'estomac, une très jeune larve au début de l'intestin grêle d'un de nos animaux, d'autres, observées dans le cæcum de deux autres, étaient plus évoluées. Aucune larve n'a été trouvée dans l'intestin postérieur.

Pendant quelque temps, tous les rats de notre élevage ont été parasités par Syphacia muris; aucune infestation aussi importante que celles signalées par W. Stahl (1961) n'a été décelée chez eux.

Comme les auteurs qui nous ont précédés, nous constatons que l'habitat principal de ce Nématode, celui où se rencontrent les mâles adultes et les femelles jusqu'au stade gravide, est le cæcum des rats de laboratoire. Ces dernières gagnent ensuite l'anus; d'après ce que nous avons observé directement, elles peuvent accomplir cette migration en rampant le long de la paroi du gros intestin. W. Stahl (1963) pense qu'elles sont plus souvent entraînées par les résidus de la digestion; c'est ce qui a dû se passer pour les femelles gravides, que nous avons trouvées au début de son observation dans les déjections du rat 752, et pour celle, encore jeune, récoltée dans l'intestin postérieur du Rongeur 747.

La plupart des œufs paraissent évoluer peu au niveau de l'anus. Chez un de nos animaux $\left(\mathrm{n}^{\circ} 747\right)$, nous apportons par deux fois la preuve (œuf embryonné dans l'estomac, très jeune larve au début du grêle), que les rats se contaminent, en ingérant des œufs de Syphacia muris arrivés à maturité. La présence de coques vides sur des préparations du prélèvement anal peut soulever la question de la pénétration par l'anus de larves écloses près de cet orifice et de leur remontée le long du gros intestin, telle qu'elle est admise par W. Schüffner (1949) chez Enterobius vermicularis de l'homme, et, avec certaines réserves, par M. J. R. Prince (1950) et par W. Stahl (1963) dans le cas de Syphacia muris du rat. Nous pensons, avec A.-G. Chabaud $(1955,1965)$, que cette modalité ne doit pas être retenue, aucune larve d'Oxyuride n'ayant été trouvée dans l'intestin postérieur de nos Rongeurs.

Jusqu'au 24 octobre, la technique de Graham a décelé des œufs de Syphacia chez tous nos rats de laboratoire soumis à cette épreuve. Nous avons été très étonnés qu'à partir de cette date, les prélèvements anaux, sauf un le 28 , aient été tous négatifs ; ils concernaient des animaux non encore testés, mais trois fois des Rongeurs antérieurement reconnus parasités; chez deux de ces derniers, des examens répétés quatre fois par semaine sont restés négatifs juqu'au 20 novembre. Faute d'avoir pu prévoir cette désinfestation, nous ignorons le déterminisme de la ponte du Nématode au moment où elle s'est produite. De toute manière, dans le cas de la souche de $S$. muris que nous avons observée, tout semble indiquer que l'évolution chez un Rongeur d'un nombre défini de générations détermine chez lui l'immunité vis-à-vis d'infestations ultérieures par cet Oxyuride. I.es circonstances ne nous ont pas permis de rechercher la nature de cette immunité. 


\section{Bibliographie}

Chabaud (A.-G.), 1955. - Essai d'interprétation phylétique des cycles évolutifs chez les Nématodes de Vertébrés. Ann. Parasitol., 30 (1-2): 83-126.

一, 1965. - Ascaridida in P. P. Grassé. Traité de Zoologie, 4 (3) : 932-1025.

Chan (K. F.), 1952. - Life cycle studies on the Nematode Syphacia obvelata. Amer. Journ. Hyg., 56: 14-21.

Hussey (K. L.), 1956. - Syphacia muris and its distribution. Journ. Parasitol., 42 (4 sect. 2) : 13-14.

Hussey (K. L.), 1957. - Syphacia muris vs. S. obvelata in laboratory rat and mice. Journ. Parasitol., 43 (5) : 555-559.

Noble (G. A.), 1965. - Groundsquirrel pinnworm infection. Journ. Parasitol., 51 (2) : 267.

Prince (M. J. R.), 1950. - Studies on the life cycle of Syphacia obvelata, a common Nematode parasite of rats. Science, 111 (2873): 66-67.

Roman (E.), 1951. - Etude écologique et morphologique sur les Acanthocéphales et les Nématodes parasites des rats de la région lyonnaise. Mem. Muséum Hist. Nat., A Zool., 2 (1) : 49-270.

-, 1957. - Modification du procédé de Telemann pour la concentration des éléments parasitaires dans les selles riches en débris cellulosiques. Bull. Assoc. Dipl. Microbiol., Nancy (68) : 15-19.

SCHüFFNER (W.), 1949. - Retrograde Oxyuren Infektion « Retrofektion ». Centralbl. Bakt., I Abt., Orig., 154 (3-5) : 220-234.

Stahl (W.), 1961. - Syphacia muris, the rat pinnworm. Science, 133 (3452): 576-577.

-, 1963. - Studies on the life cycle of Syphacia muris, the rat pinnworm. Keio Journ. Med., 12 (2) : 55-61.

TINER (J. D.), 1948. - Syphacia eutamii n. sp. from the least Chipmunk Eutamias minimus, with a key to the genus (Nematoda Oxyuridae). Journ. Parasitol., 51 (2): 87-92.

YAMAGUTI (S.), 1935. - Studies, on the helminth fauna of Japan, part 13, Mammalian Nematodes. Japan Journ. Zool., 6: 453-457. 